\title{
Isolation and characterization of bacterium producing lipid from short-chain fatty acids
}

Yoshiko Okamura ${ }^{1,4} *$, Shota Nakai ${ }^{1}$, Masahiko Ohkawachi ${ }^{1}$, Masahiro Suemitsu ${ }^{1}$, Hirokazu

Takahashi ${ }^{1,4}$, Tsunehiro Aki ${ }^{1,4}$, Yukihiko Matsumura ${ }^{2,4}$, Takahisa Tajima ${ }^{1,4}$, Yutaka

Nakashimada $^{1,4}$ and Mitsufumi Matsumoto ${ }^{3}$.

${ }^{1}$ Department of Molecular Biotechnology, Graduate School of Advanced Sciences of Matter, Hiroshima University, 1-3-1 Kagamiyama, Higashi-Hiroshima 739-8530, Japan ${ }^{2}$ Division of Energy and Environmental Engineering, Institute of Engineering, Hiroshima University, 1-4-1

Kagamiyama, Higashi-Hiroshima 739-8527, Japan

${ }^{3}$ Biotechnology Laboratory, Electric Power Development Co., Ltd., Yanagisaki-machi, Wakamatsu-ku, Kitakyusyu 808-0111, Japan

${ }^{4}$ CREST, JST, Sanbancho 5, Chiyoda-ku, Tokyo 102-0075, Japan

* Corresponding author.

TEL/FAX: +81 824244583 .

E-mail address: okamuray@hiroshima-u.ac.jp 


\begin{abstract}
Anaerobic fermentation generates propionic acid, which inhibits microbial growth and accumulates in wastewater containing increased amounts of organic matter. We therefore isolated a propionic acid-assimilating bacterium that could produce triacylglycerol, for use in wastewater treatment. Nitratireductor sp. strain OM-1 can proliferate in medium containing propionic, acetic, butyric, and valeric acids as well as glycerol, and produces triacylglycerol when both propionic and acetic acids or glycerol are present. In composite model wastewater containing acetic acid, propionic acid and glycerol, this strain shows an even higher conversion rate, suggesting that it is suitable for wastewater treatment. Further, nitrogen depletion in medium containing an acetic-propionic acid mixture resulted in the production of the light oil 2-butenoic acid 1-methylethyl ester, but not triacylglycerol. Collectively, our data indicate that strain OM-1 has the potential to reduce accumulation of activated sludge in wastewater treatment and may contribute to the production of biodiesel.
\end{abstract}

Keywords: biodiesel, light oil, propionic acid, triacylglycerol production, wastewater treatment 


\section{Introduction}

The activated sludge process, which involves the microbial degradation of organic matter in an aeration tank, is commonly used for wastewater disposal. Microbes in activated sludge consume the organic matter, nitrogen, and phosphorus in wastewater, and half of the organic mass is converted into excess sludge. This excess sludge is concentrated, dehydrated, and may be incinerated, after which, it is eventually disposed of. However, this is problematic because the majority of the energy and expense required for the activated sludge process goes towards the disposal of the excess sludge. Therefore, it is imperative that both the generation of excess sludge and the costs of disposal should be reduced.

A great deal of ongoing research has focused on efforts to minimize excess sludge (Wei et al. 2003), using processes such as ozonation (Yasui et al. 1996, Sakai et al. 1997, Kamiya and Hirotsuji 1998), chlorination (Saby et al. 2002), thermal treatment (Canales et al. 1994), thermo-chemical treatment (Rocher et al. 2001), and chemical uncouplers that impede microbial growth (Low and Chase 1998, Strand et al. 2003, Yang et al. 2003). Furthermore, considerable work is currently aimed at avoiding excess sludge production through bioconversion. Examples of this include the re-use of waste-activated sludge by hydrolyzation and cultivation of microalgae using the hydrolysate for lipid production (Wen et al. 2013), as well as the removal of chemical oxygen demand (COD) in wastewater by treatment with carotenoid producing bacteria (Zhou et al. 2014). Among such attempts, anaerobic digestion has been widely used to reduce and stabilize excess activated sludge, and it is currently in practical use (Mata-Alvarez et al. 2000). Further, because this technology can recover methane as an end-product, it is a potential process for the recovery of renewable energy (Gunaseelan 1997).

In anaerobic digestion, methane is produced from organic substances in a three-step process, involving hydrolysis/acidogenesis, acetogenesis and methanogenesis. In the first 
step, organic substances are converted to volatile fatty acids (VFAs) by various bacteria. In the second step, VFAs other than acetate, such as propionate and butyrate, are oxidized to acetate and hydrogen by propionate- and butyrate-oxidizing bacteria, respectively. In the final step, methane is produced from hydrogen and carbon dioxide by hydrogenotrophic methanogens, and from acetate by acetoclastic methanogens. The sequential conversion of organic substances to methane is well-organized, with a low organic loading rate during anaerobic digestion, while VFAs accumulate with increased organic loading rate (Kida et al. 1990). The accumulation of VFAs should be avoided, as they impede the growth of methanogenic bacteria and many other microbes (Nanba et al. 1983). Furthermore, VFAs remaining in the methane fermentation residue may act as a substrate for the production of useful materials, such as fuel. In this context, the development of a two-step process for the treatment of excess activated sludge, in which the fermentation residue is processed using bacteria that convert VFAs to useful bio-products, should be feasible for the high-rate processing of excess sludge with a high organic loading rate.

In this study, we aimed to isolate a bacterium capable of converting VFAs, particularly acetic and propionic acids, which mainly accumulate with increased organic loading rate (Kida et al. 1990), directly into triacylglycerol (TAG). We focused on seawater as our microbial source, as marine biodiesel producers have several advantages in biofuel production strategies (Matsumoto et al. 2010). Here, we report lipid production from VFAs by a newly isolated marine bacterium, which we have named Nitratireductor sp. strain OM-1.

\section{Materials and Methods}

\subsection{Screening and isolation of a propionic acid-assimilating bacterium}


Seawater, including gravel gathered from the shores of Osaki Shimojima Island, Hiroshima, was used as a microbial source, and this was enriched at room temperature in ATCC medium \#1409 (1409 medium, http://www.atcc.org/ /media/27EC6C142C974F408D98282263B756C6.ashx), containing $1.0 \mathrm{~g} / \mathrm{L}$ sodium acetate (approximately $10 \mathrm{mM}$ ), and supplemented with $10 \mathrm{mM}$ propionic acid. Enriched bacteria were then seeded onto 1409 medium plates, containing $10 \mathrm{mM}$ propionic acid and solidified with $1 \%$ agar, and these were overlaid with $0.7 \%$ agar. Cultivation was carried out at room temperature (approximately $20 \sim 26^{\circ} \mathrm{C}$ ) in the dark. The resulting colonies were inoculated into $3 \mathrm{~mL}$ of 1409 medium, containing $10 \mathrm{mM}$ propionic acid in test tubes, and these cultures were incubated at $28^{\circ} \mathrm{C}$ in the dark with shaking at 100 rpm under aerobic conditions. Candidate strains were selected based on high lipid production, as assessed by nile red staining.

\subsection{Cultivation}

For assimilation testing, acetic acid, propionic acid, butyric acid, valeric acid, or glycerol was used as the sole carbon source, at a concentration of $10 \mathrm{mM}$, in 1409 medium; in these experiments, sodium acetate was omitted. Liquid cultures were incubated at $28^{\circ} \mathrm{C}$ in the dark with shaking at $100 \mathrm{rpm}$. Cultures for calculating the conversion rate of each carbon source into total lipids were grown in a total volume of $300 \mathrm{~mL}$ in 500-mL Erlenmeyer flasks. These were incubated at $28^{\circ} \mathrm{C}$ in the dark with shaking at $100 \mathrm{rpm}$ for 7 days. For nitrogen starvation assays, the concentration of ammonium chloride in 1409 medium was varied from 0 to $10 \mathrm{mM}$.

\subsection{Lipid extraction and methyl esterification}

Three 300-mL cultures, totaling $900 \mathrm{~mL}$ of bacterial culture, were used for the calculation 
of conversion rates. Bacteria were harvested, washed with PBS, and lyophilized. Subsequently, $3 \mathrm{~mL}$ of hexane was added to the dried cells, and these were thoroughly vortexed according to Matsumoto et al. (2010). This step was repeated two additional times until a total extract volume of $9 \mathrm{~mL}$ was obtained. After centrifugation at 5,000 x g for $10 \mathrm{~min}$, the hexane extract was transferred to a test tube and desiccated by nitrogen gas flow $\left(60^{\circ} \mathrm{C}\right)$. Resulting tubes were then weighed to estimate total lipid amount.

For lipid content analysis, the extraction method was modified based on the Folch et al. (1957) to be suitable for bacterial cells. Total lipid was extracted from the dried cells with $500 \mu \mathrm{L}$ of chloroform/methanol (2:1, v/v), and this was repeated twice to obtain a total extract volume of $1.5 \mathrm{~mL}$. After an additional $500 \mu \mathrm{L}$ of milliQ water was added, the extract was vortexed and centrifuged at 5,000 x g for $10 \mathrm{~min}$. The lower chloroform phase was then transferred to a test tube and desiccated by nitrogen gas flow $\left(60^{\circ} \mathrm{C}\right)$, after which, $25 \mu \mathrm{L}$ of chloroform was added to extract total lipids.

For fatty acid measurement, $1 \mathrm{~mL}$ of $10 \%$ hydrochloric acid-methanol was added to the total lipid extract, and this was heated at $60^{\circ} \mathrm{C}$ for $2 \mathrm{~h}$ to methyl esterify the fatty acids. The sample was then completely desiccated by nitrogen gas flow $\left(60^{\circ} \mathrm{C}\right)$, after which $2 \mathrm{~mL}$ of hexane and $1 \mathrm{~mL}$ of distilled water were added, and the water-soluble component (e.g., glycerol) was dissolved in the aqueous phase. Samples were then centrifuged at 10,000 $\mathrm{x} \mathrm{g}$ for $10 \mathrm{~min}$, and the hexane phase was transferred to a new test tube and desiccated by nitrogen gas flow $\left(60^{\circ} \mathrm{C}\right)$. After desiccation, $10 \mu \mathrm{L}$ of hexane was added, and fatty acid analysis was performed.

\subsection{Analytical methods}

Total lipids were weighed after evaporation to dryness. Lipid content was analyzed by gas chromatography (GC-17A, CBM-102, both Shimadzu Corp. Tokyo, Japan), using a 
DB-5HT capillary GC column (Agilent Technologies, California, USA). The conditions for gas chromatography analysis were, as follows: column oven, $100^{\circ} \mathrm{C}$ to $380^{\circ} \mathrm{C}$ (increasing at $10^{\circ} \mathrm{C} / \mathrm{min}$ ); carrier gas, $\mathrm{N}_{2}$; sample volatilization chamber, $300^{\circ} \mathrm{C}$; detector, $380^{\circ} \mathrm{C}$; pressure, $107 \mathrm{kPa}$; total flow rate, $16 \mathrm{~mL} / \mathrm{min}$; column flow rate, $1.19 \mathrm{~mL} / \mathrm{min}$; and split ratio, 1:10. GCSolution software, version 2.4 (Shimadzu Corp.) was used for the analysis.

Fatty acids were analyzed by gas chromatography (GC-2014, Shimadzu Corp.), using a TC-70 capillary GC column (GL Sciences Inc. Tokyo, Japan). The conditions for gas chromatography analysis were as follows: column oven, $180^{\circ} \mathrm{C}$; sample volatilization chamber, $270^{\circ} \mathrm{C}$; entrance pressure, $105 \mathrm{kPa}$; total flow rate, $20.3 \mathrm{~mL} / \mathrm{min}$; and split ratio, 1:20. Class-GC10 software (Shimadzu Corp.), was used for the analysis. The fatty acid standard was a 37-Component FAME Mix on the SPB-PUFA (Supelco, Pennsylvania, USA) and the fatty acid peaks in the chromatograms were identified by comparison with the retention times of the standards.

Acetic acid, propionic acid, and butyric acid were quantified by high-performance liquid chromatography (HPLC) using an ELITE LaChrom instrument (Hitachi, Tokyo, Japan), equipped with an InertSustain C18 column (GL Sciences Inc.) maintained at $40^{\circ} \mathrm{C}$. Phosphoric acid $(0.5 \%, \mathrm{v} / \mathrm{v})$ was used as the mobile phase at a flow rate of $1.0 \mathrm{~mL} / \mathrm{min}$. Valeric acid was measured by HPLC as described by Miura et al. (2014), and glycerol was measured using the Glycerol Assay Kit (Cayman Chemical Company, Michigan, USA).

Bacterial growth was evaluated by measuring the OD660 of the culture using an ANA-18A+ Model Colorimeter (Tokyo Koden, Japan). Nile red fluorescence was measured using a BX60 upright microscope system (Olympus, Tokyo, Japan) and BX-FLA epi illumination fluorescence device (Olympus). Fluorescence was observed using a BP520-550 filter and IG excitation.

A fragment of the 16S rRNA gene was amplified by direct PCR with the $16 \mathrm{~S}$ universal 
primers 8F (5’-AGAGTTTGATCCTGGCTCAG-3’) and 536R

(5’-GWATTACCGCGGCKGCTG-3'), using 1- $\mu \mathrm{L}$ aliquots of bacterial culture as template.

The PCR reaction solution was prepared according to the protocol recommended for PrimeSTAR HS DNA polymerase (Takara Bio, Shiga, Japan). PCR reactions were carried out with initial denaturation at $94^{\circ} \mathrm{C}$ for $1 \mathrm{~min}$, followed by 30 cycles of denaturation at $98^{\circ} \mathrm{C}$ for $10 \mathrm{~s}$, annealing at $56^{\circ} \mathrm{C}$ for $5 \mathrm{~s}$, and elongation at $72^{\circ} \mathrm{C}$ for $30 \mathrm{~s}$, followed by a final elongation at $72^{\circ} \mathrm{C}$ for $1 \mathrm{~min}$. The PCR products were purified using a GEL/PCR Purification Column (Favorgen Biotech Corporation, Ping-Tung, Taiwan) and then cloned using a Zero Blunt TOPO PCR Cloning Kit (Invitrogen, California, USA). The sequences of the resulting clones were analyzed by Eurofins Genomics Co., Ltd (Tokyo, Japan).

\section{Results and Discussion}

\subsection{Isolation of bacteria based on lipid production}

Approximately 100 colonies grown on 1409 medium agar containing acetic and propionic acids were individually cultivated in liquid medium. Lipid synthesis was assessed by nile red staining, and five candidates were selected. Partial 16S rRNA gene sequences were determined, and we found that four of these five cultures contained several distinct sequences, indicating they were non-axenic. From the remaining candidate, only one sequence was amplified, which exhibited 99\% homology to the 16S rRNA gene of Nitratireductor aquimarinus (Jang et al. 2011). Thus, we designated this new bacterium as Nitratireductor sp. strain OM-1 and characterized this isolate in further experiments.

\subsection{Growth of Nitratireductor sp. OM-1 in VFAs and glycerol}

The strain OM-1 grew in ATCC 1409 medium containing $10 \mathrm{mM}$ each of acetic and 
propionic acids and reached an $\mathrm{OD}_{660}$ of 0.66 after $120 \mathrm{~h}$ of incubation (Fig. 1A). Lipid production increased with growth and reached $8.8 \mathrm{mg} / \mathrm{L}$ at $120 \mathrm{~h}$ (Fig. 2A). Of note, this is the first report of the bacterium that can convert VFAs such as acetic acid and propionic acids into lipid, suggesting that Nitratireductor sp. OM-1 is a promising candidate, not only for reducing excess VFAs remaining after anaerobic digestion, but also for producing raw materials for biodiesel.

In addition to acetic and propionic acids, anaerobic digestion produces other VFAs, such as butyric and valeric acids. To evaluate whether Nitratireductor sp. OM-1 can assimilate other VFAs produced during anaerobic digestion, this strain was cultivated with each VFA as the sole carbon source, and both growth and lipid production were assessed. With VFAs ranging from $\mathrm{C} 2$ to $\mathrm{C} 5$ as the sole carbon source, strain OM-1 grew under all conditions, with steady-state values that approached $\mathrm{OD}_{660}=0.7-0.8$ in the presence of all VFAs except propionate (Fig. 1). Acetic acid was the most preferred organic acid and was fully consumed within $72 \mathrm{~h}$ (Fig. 1B). Butyric acid, at a concentration of approximately $6 \mathrm{mM}$, was also consumed (Fig. 1D). Interestingly, valeric acid was consumed slowly, at a steady rate (Fig. 1E). Conversely, in medium containing propionic acid, the assimilation rate was low, and growth stopped at OD660 $=0.45$ (Fig. 1C). However, it is important to note that propionic acid was fully consumed when mixed with acetic acid (Fig. 1A), suggesting that the presence of acetic acid accelerates the full assimilation of propionic acid. Because production of biodiesel from TAG gives rise to waste glycerol, the assimilation of glycerol was also examined. Notably, we found that the rate of glycerol consumption was second only to that of acetic acid, indicating that glycerol is a favorable substrate for this organism (Fig. 1F). We therefore suggest that it may be possible to recycle waste glycerol to produce TAG using the strain OM-1. 


\subsection{Lipid production from VFAs and glycerol by Nitratireductor sp. OM-1}

We next analyzed the lipid content of Nitratireductor sp. OM-1 cultures containing each of the various VFAs, described above (Fig. 2). In cultures containing an acetic acidpropionic acid mixture (Fig. 2A), acetic acid (Fig. 2B), or glycerol (Fig. 2F), lipids were produced concurrent with cell proliferation, even during exponential growth. In contrast, cultures containing only propionic acid (Fig. 2C) or butyric acid (Fig. 2D) exhibited a distinct lipid-production phase between the end of exponential growth and stationary phase. The carbon yields of total lipids from the various carbon sources are listed in Table 1. It is important to note that culture conditions used to generate the data in Table 1 were not the same as those used in the experiment illustrated in Fig. 2; therefore, lipid production was not identical. However, in both cases, butyric acid was found to result in the highest lipid production and carbon yield. For propionic acid, on the other hand, lipid production was lowest, likely due to low cell mass, while carbon yield was the second highest observed.

Interestingly, the lipid constituents differed depending on the carbon source. For acetic acid-only cultures, squalene and TAG were the major lipid species; for propionic acid, it was squalene. Myristic acid was the major lipid produced from butyric acid, whereas palmitic acid was generated from valeric acid, and stearic acid and TAG were primarily produced from glycerol. The main constituents resulting from the acetic acid-propionic acid mixture were TAG, squalene, and stearic acid. It is expected that acetic acid is converted into fatty acids (FAs) through acetyl-CoA, and the resulting FAs are stored in TAG; whereas propionic acid is likely converted into odd-numbered fatty acids through propionyl-CoA, leading to squalene synthesis via C15. Notably, the TAG levels were greatest with the acetic acidpropionic acid mixture. Glycerol also exhibited a high TAG conversion rate, demonstrating that waste glycerol will also favor TAG production. 


\subsection{Lipid production in model wastewater containing glycerol}

The data shown in Fig. 1A suggest that a 1:1 mixture with acetic acid is desirable for the complete consumption of propionic acid. Therefore, model wastewater was prepared by fixing the molar concentrations of acetic acid:propionic acid at 1:1 and adding waste glycerol. We then determined the time courses for carbon consumption and lipid production within this model system (Fig. 3). In this assay, Nitratireductor sp. OM-1 first utilized glycerol for growth, and propionic acid was assimilated only after depletion of glycerol (Fig. 3A). An increase in biomass was not observed during this phase, but the assimilated carbon was converted into lipids (Fig. 3B), resulting in the highest production of total lipids of all conditions tested. This suggests the presence of a synergistic effect, exceeding the respective production levels observed with acetic acid, propionic acid or glycerol alone.

To date, our previous research has focused on energy production from brown algae under high salinity conditions through methane fermentation (Miura et al. 2015). The process of methane fermentation consists of three steps: hydrolysis/acidogenesis, acetogenesis and methanogenesis. In the first step, the brown algae are converted primarily to acetate and propionate (Miura et al. 2014). Therefore, the results of the present study involving Nitratireductor sp. OM-1 illustrate an alternative method for methane or biodiesel production from brown algae.

\subsection{Effect of nitrogen depletion on lipid production by Nitratireductor sp. OM-1}

Based on a study investigating the effect of nitrogen deficiency on lipid production (Li et al. 2008), the amount of ammonium chloride in 1409 medium was varied in stages, using concentrations of $10,1,0.1$, and $0 \mathrm{mM}$, and cultivation was carried out for 1 week. The bacteria were then harvested, stained with nile red, and observed using fluorescence microscopy. The concentration of ammonium chloride in the original medium is about 18.5 
$\mathrm{mM}$. When bacteria were grown in $1,0.1$ or $0 \mathrm{mM}$ ammonium chloride, the fluorescence was strong, particularly in the complete absence of ammonium chloride $(0 \mathrm{mM})$, where the lipid content was calculated to reach 70\% (Table 2). The yellow glow often observed with nile red staining is due to neutral lipids such as triglycerides (Diaz et al. 2008). Interestingly, although we predicted a large accumulation of TAG, it was converted to 2-butenoic acid 1-methylethyl ester at lower nitrogen concentrations, particularly in media with $0 \mathrm{mM}$ ammonium chloride, where this ester content accounts for $50 \%$ of the total lipid. Moreover, under these conditions, fatty acids account for about $4 \%$ of the total lipids, and the main components of methyl esterified fatty acids and glycerides were linoleic acid, palmitoleic acid and pentadecenoic acid (Table 2). 2-Butenoic acid 1-methylethyl ester is a combustible substance, and the heat of combustion for its derivative, 2-butenoic acid, is $2.00 \mathrm{MJ} / \mathrm{mol}$ (https://pubchem.ncbi.nlm.nih.gov/compound/crotonic acid\#section=Top), which is higher than the heat of combustion for ethanol, which is $1336.8 \mathrm{~kJ} / \mathrm{mol}$ at $25^{\circ} \mathrm{C}$ (https://pubchem.ncbi.nlm.nih.gov/compound/702\#section=Top). These results indicate that nitrogen deficiency promotes the production of light oils rather than TAG.

\section{Conclusions}

We isolated the bacterium, Nitratireductor sp. OM-1, which can produce lipids from lower organic acids in treated wastewater. This strain proliferates in propionic, acetic, butyric or valeric acid-containing medium and primarily produces TAG in medium containing acetic and propionic acids or glycerol. Growth in composite model wastewater resulted in a higher conversion rate than observed with individual carbon sources and nitrogen depletion in acetic and propionic acid-containing medium produced 2-butenoic acid 1-methylethyl ester. Our data suggest Nitratireductor sp. OM-1 is well-suited for lower organic acid wastewater treatment as it can reduce activated sludge accumulation and may 
contribute to biodiesel production.

\section{Acknowledgements}

This work was supported by the Core Research of Evolutional Science and Technology program (CREST) from the Japan Science and Technology Agency (JST). 


\section{References}

1. Canales, A., Pareilleux, A., Rols, J., Goma, G., Huyard, A., 1994. Decreased sludge production strategy for domestic waste- water treatment. Water Sci. Technol. 30, 97-106.

2. Diaz, G., Melis, M., Batetta, B., Angius, F., Falchi, A.M., 2008. Hydrophobic characterization of intracellular lipides in situ by Nile Red red/yellow emission ratio. Micron 39, 819-824.

3. Folch, J., Lees, M., Sloane Stanley, G.H., 1957. A simple method for the isolation and purification of total lipids from animal tissues. J. Biol. Chem. 226, 497-509.

4. Gunaseelan, V.N., 1997. Anaerobic digestion of biomass for methane production. A review. Biomass Bioener 13, 83-114.

5. Jang, G.I., Hwang, C.Y., Cho, B.C., 2011. Nitratireductor aquimarinus sp. nov., isolated from a culture of the diatom Skeletonema costatum, and emended description of the genus Nitratireductor. Int. J. Syst. Evol. Microbiol. 61, 2676-2681.

6. Kamiya, T., Hirotsuji, J., 1998. New combined system of biological process and intermittent ozonation for ad- vanced wastewater treatment. Water Sci. Technol. 38, 145- 53.

7. Kida, K., Morimura, S., Sonoda, Y., Ohbe, M., Tanemura, K., 1990. Treatment of wastewater from a shouchu distillery by an anaerobic fluidized-bed reactor. J. Brew. Soc. Japan 85, 651-656.

8. Li, Y., Horsman, M., Wang, B., Wu, N., Lan, C.Q., 2008. Effects of nitrogen sources on cell growth and lipid accumulation of green alga Neochloris oleoabundans. Appl. Microbiol. Biotechnol. 81, 629-636.

9. Low, E.W., Chase, H.A., 1998. The use of chemical uncouplers for reducing biomass production during biodegradation. Water Sci. Technol. 37, 399-402.

10. Matsumoto, M., Sugiyama, H., Maeda, Y., Sato, R., Tanaka, T., Matsunaga, T., 2010. Marine Diatom, Navicula sp. Strain JPCC DA0580 and Marine Green Alga, Chlorella sp. Strain NKG400014 as Potential Sources for Biodiesel Production. Appl. Biochem.

11. Biotechnol. 161, 483-490.

12. Mata-Alvarez, J., Macé, S., Llabrés, P., 2000. Anaerobic digestion of organic solid wastes. An overview of research achievements and perspectives. Bioresour. Technol. 74, 3-16.

13. Miura, T., Kita, A., Okamura, Y., Aki, T., Matsumura, Y., Tajima, T., Kato, J., Nakashimada, Y., 2014. Evaluation of marine sediments as microbial sources for methane production from brown algae under high salinity. Bioresour. Technol. 169, 362-366.

14. Miura, T., Kita, A., Okamura, Y., Aki, T., Matsumura, Y., Tajima, T., Kato, J., Nakashimada, Y., 2015. Improved methane production from brown algae under high 
salinity by fed-batch accimilation method. Bioresour. Technol. 187, 275-281.

15. Nanba, A., Nukada, R., Nagai, S., 1983. Inhibition by acetic and propionic acids of the growth of Propionibacterium shermanii. J. Ferment. Technol. 61, 551-556.

16. Rocher, M., Roux, G., Goma, G., Begue, A.P., Louvel, L., Rols, J.L., 2001. Excess sludge reduction in activated sludge processes by integrating biomass alkaline heat treatment. Water Sci. Technol. 44, 437-44.

17. Saby, S., Djafer, M., Chen, G.H., 2002. Feasibility of using a chlorination step to reduce excess sludge in activated sludge process. Water Res. 36, 656-666.

18. Sakai, Y., Fukase, T., Yasui, H., Shibata, M. 1997. An activated sludge process without excess sludge production. Water Sci. Technol. 36, 163-170.

19. Strand, S.E., Harem, G.N., Stensel, H.D., 1997. Activated-sludge yield reduction using chemical uncouplers. Water Environ. Res. 71, 454-458.

20. Wei, Y., Van Houten, R.T., Borger, A.R., Eikelboom, D.H., Fan, Y., 2003.

Minimization of excess sludge production for biological wastewater treatment. Water Res. 37, 4453- 4467.

21. Wen, Q., Chen, Z., Li, P., Duan, R., Ren, N., 2013. Lipid production for biofuels from hydrolyzate of waste activated sludge by heterotrophic Chlorella protothecoides. Bioresour. Technol. 143, 695-698.

22. Yasui, H., Nakamura, K., Sakuma, S., Iwasaki, M., Sakai, Y., 1996. A full-scale operation of a novel activated sludge process without excess sludge production. Water Sci. Technol. 34, 395-404.

23. Yang, X.F., Xie, M.L., Liu, Y., 2003. Metabolic uncouplers reduce excess sludge production in an activated sludge process. Process Biochem. 38, 1373-1377.

24. Zhou, Q., Zhang, P., Zhang, G., 2014. Biomass and carotenoid production in photosynthetic bacteria wastewater treatment: Effects of light intensity. Bioresour. Technol. 171, 330-335. 


\section{Figure Captions}

Fig. 1. Growth of Nitratireductor sp. OM-1 in various carbon sources. Carbon sources are as follows: (A) a mixture of $10 \mathrm{mM}$ acetic acid and $10 \mathrm{mM}$ propionic acid as standard, (B) $10 \mathrm{mM}$ acetic acid, (C) $10 \mathrm{mM}$ propionic acid, (D) $10 \mathrm{mM}$ butyric acid, (E) $10 \mathrm{mM}$ valeric acid, and (F) $8 \mathrm{mM}$ glycerol. Solid lines indicate cell growth, and dashed lines show consumption of the carbon source. Symbols are as follows: closed diamond, acetic acid; closed triangle, propionic acid; closed circle, butyric acid; open circle, valeric acid; open diamond, glycerol.

Fig. 2. Time course of lipid accumulation by Nitratireductor sp. OM-1 grown in the presence of various carbon sources. Carbon sources are as follows: (A) a mixture of $10 \mathrm{mM}$ acetic acid and $10 \mathrm{mM}$ propionic acid as standard, (B) $10 \mathrm{mM}$ acetic acid, (C) $10 \mathrm{mM}$ propionic acid, (D) $10 \mathrm{mM}$ butyric acid, (E) $10 \mathrm{mM}$ valeric acid, and (F) $8 \mathrm{mM}$ glycerol. Solid line indicates lipid production, and the bars indicate lipids produced: green, squalene; red, triacylglycerol; and blue, other lipids.

Fig. 3. Nitratireductor sp. OM-1 growth (A) and lipid production (B) in model wastewater. Acetic acid, propionic acid, and glycerol (10 mM each) were added to ATCC 1409 medium without sodium acetate to generate the model wastewater. Solid line indicates bacterial growth curve, and dashed line shows carbon consumption; Symbols are as follows: closed diamond, acetic acid; closed triangle, propionic acid; and open diamond, glycerol. Bars indicate lipids produced: green, squalene; red, triacylglycerol; and blue, other lipids. 
(A)
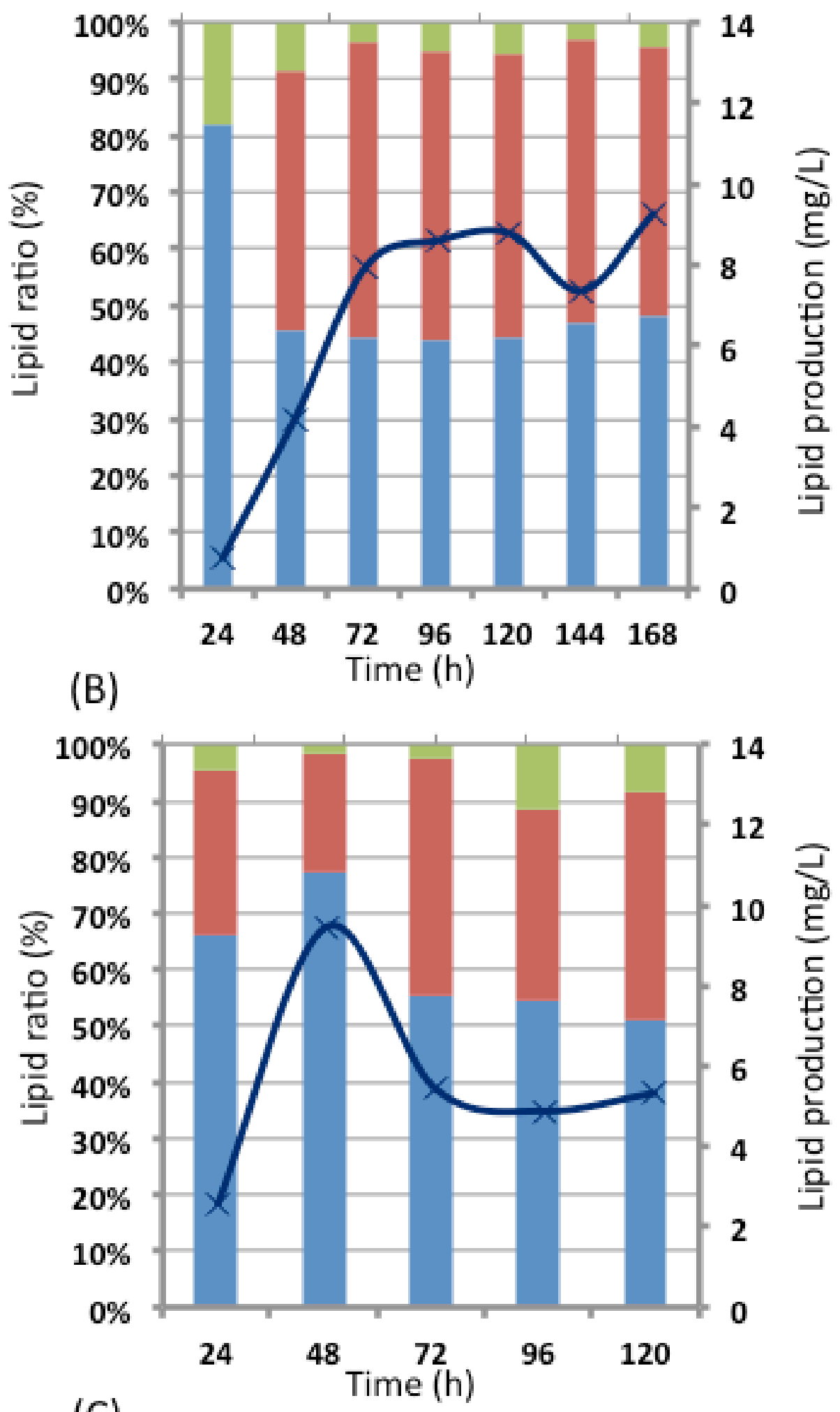

(C)

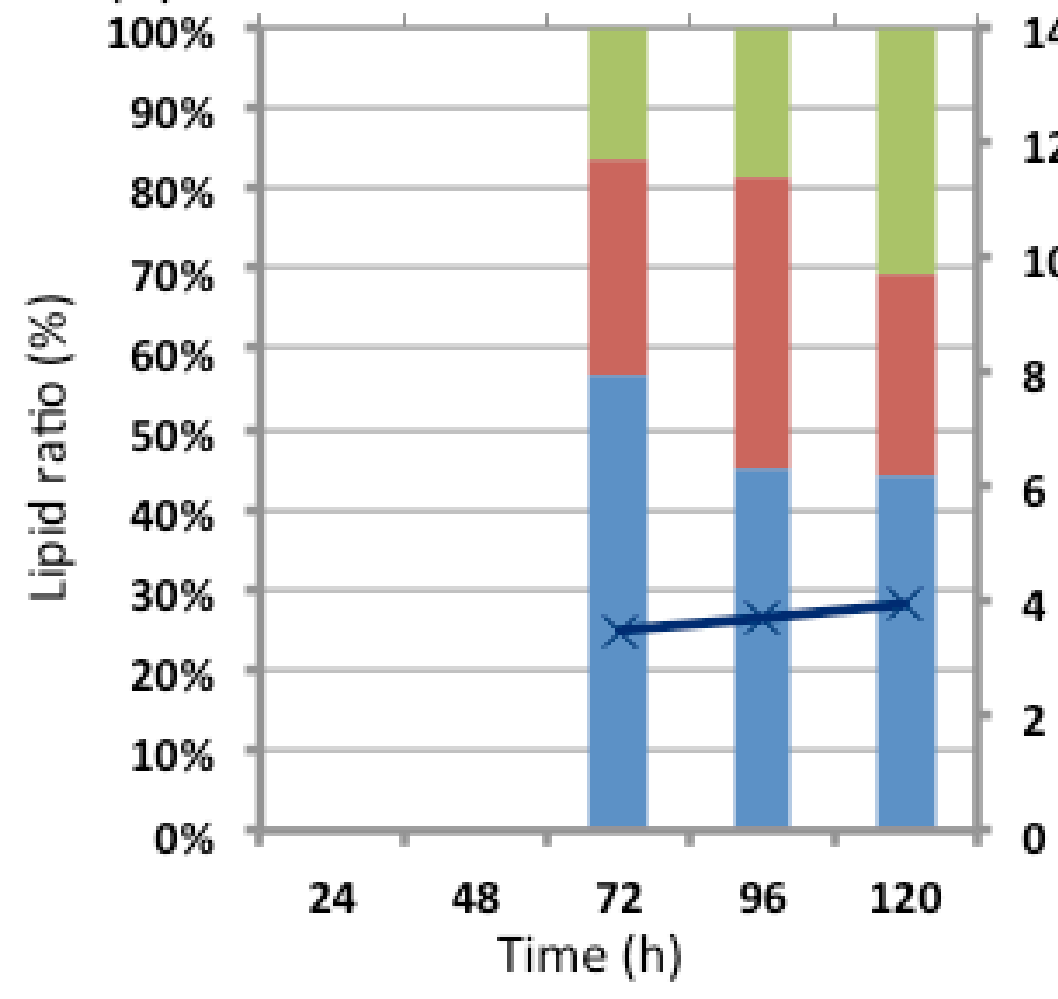

(D)
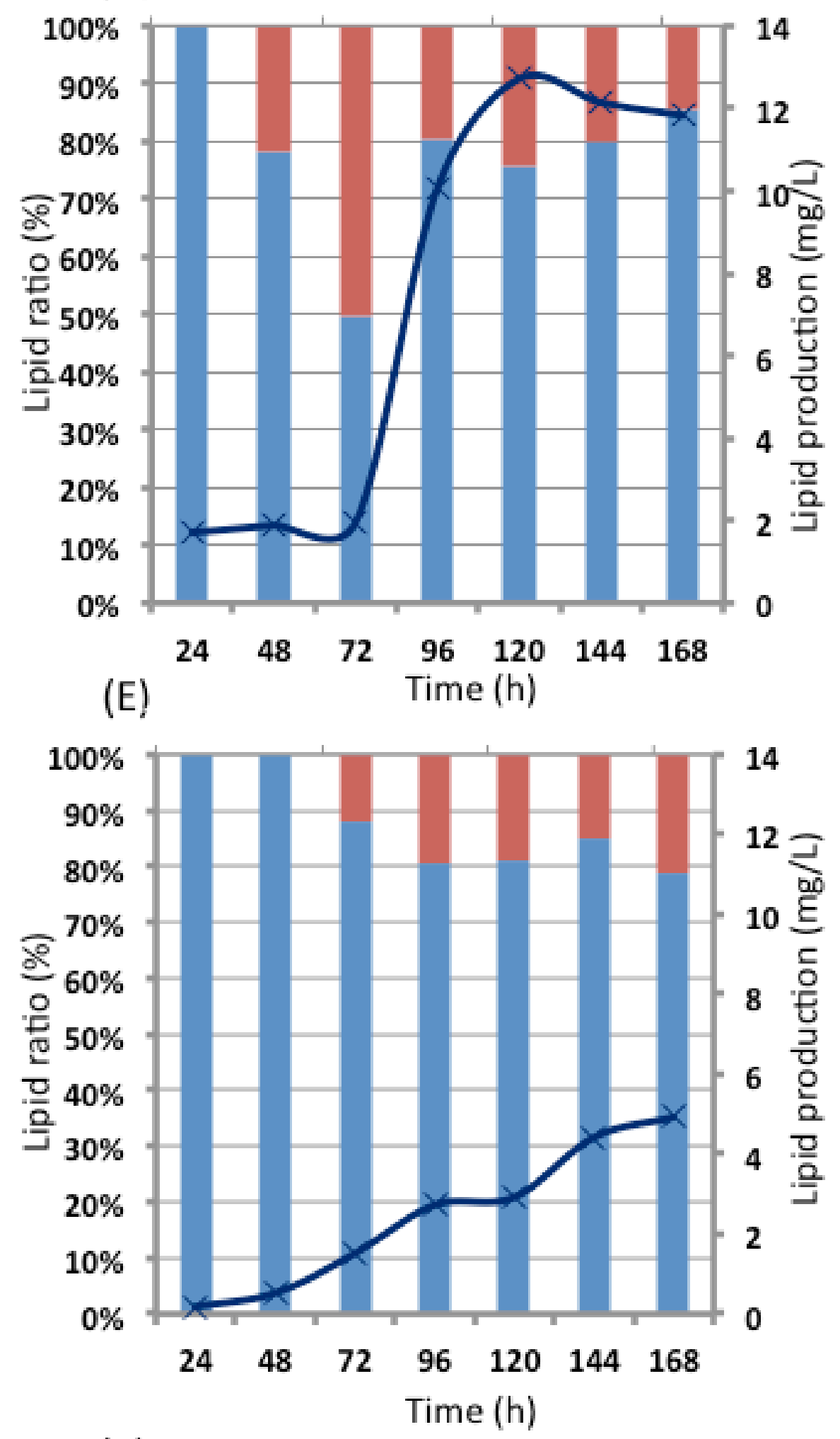

(F)

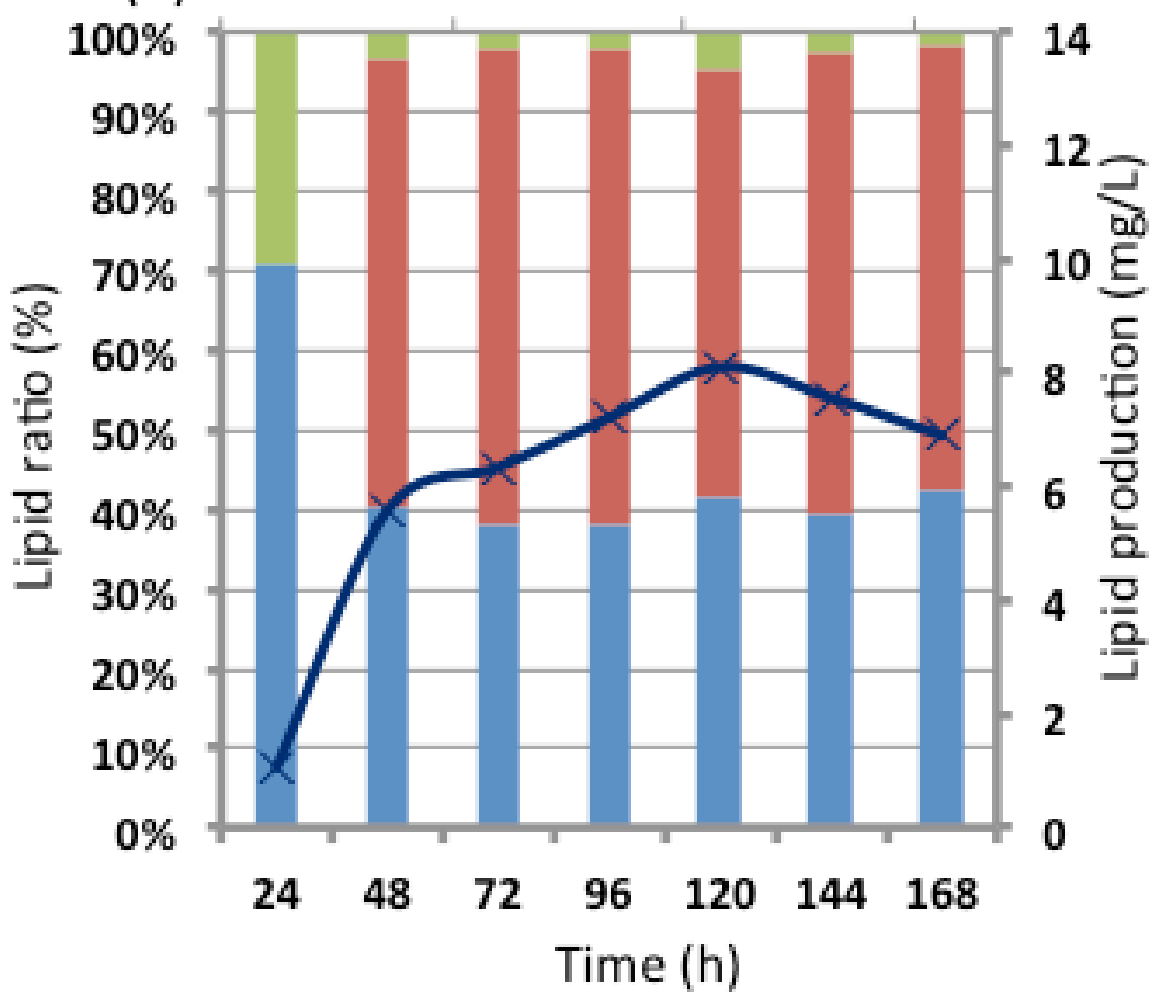

Fig. 2 


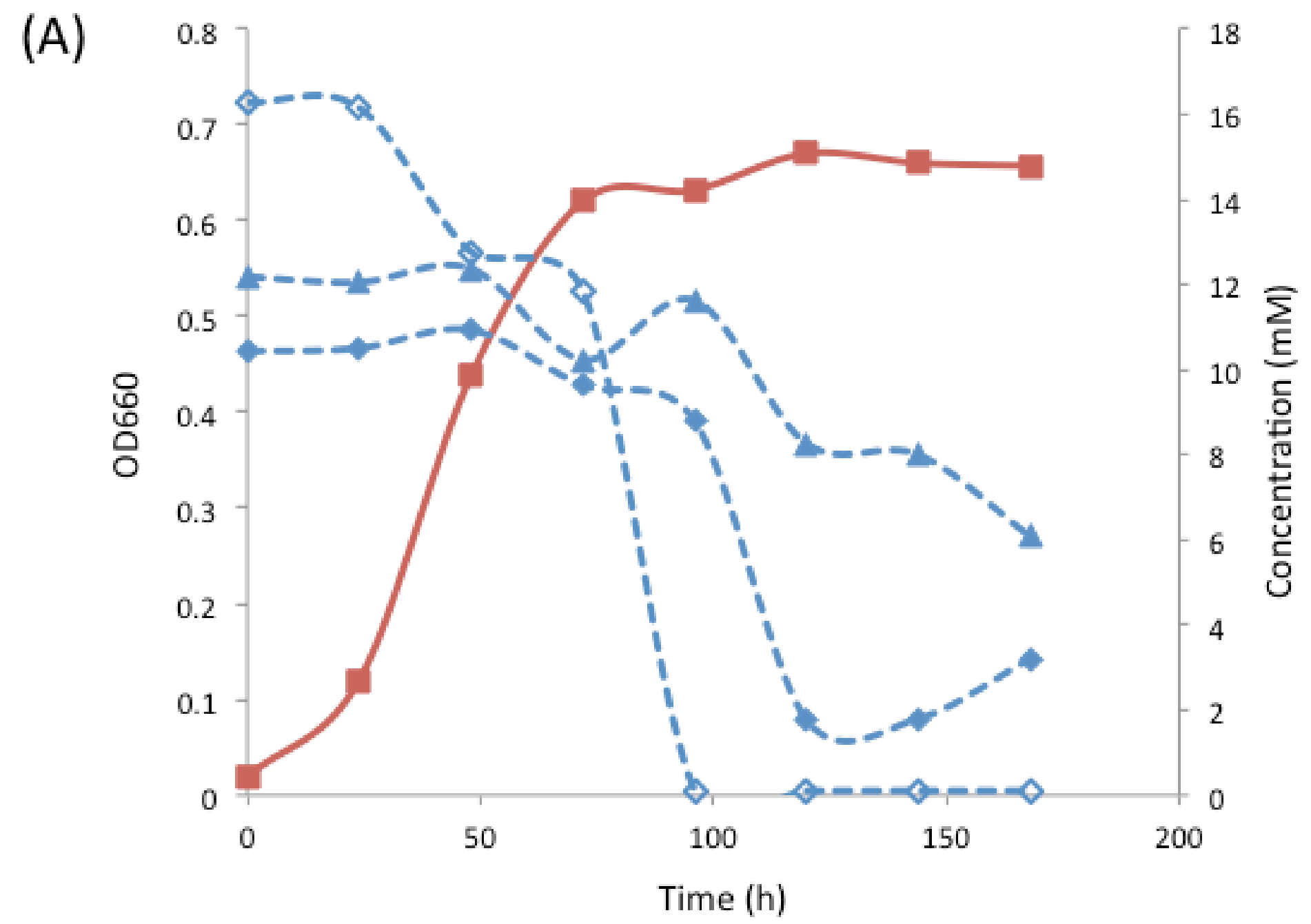

(B)

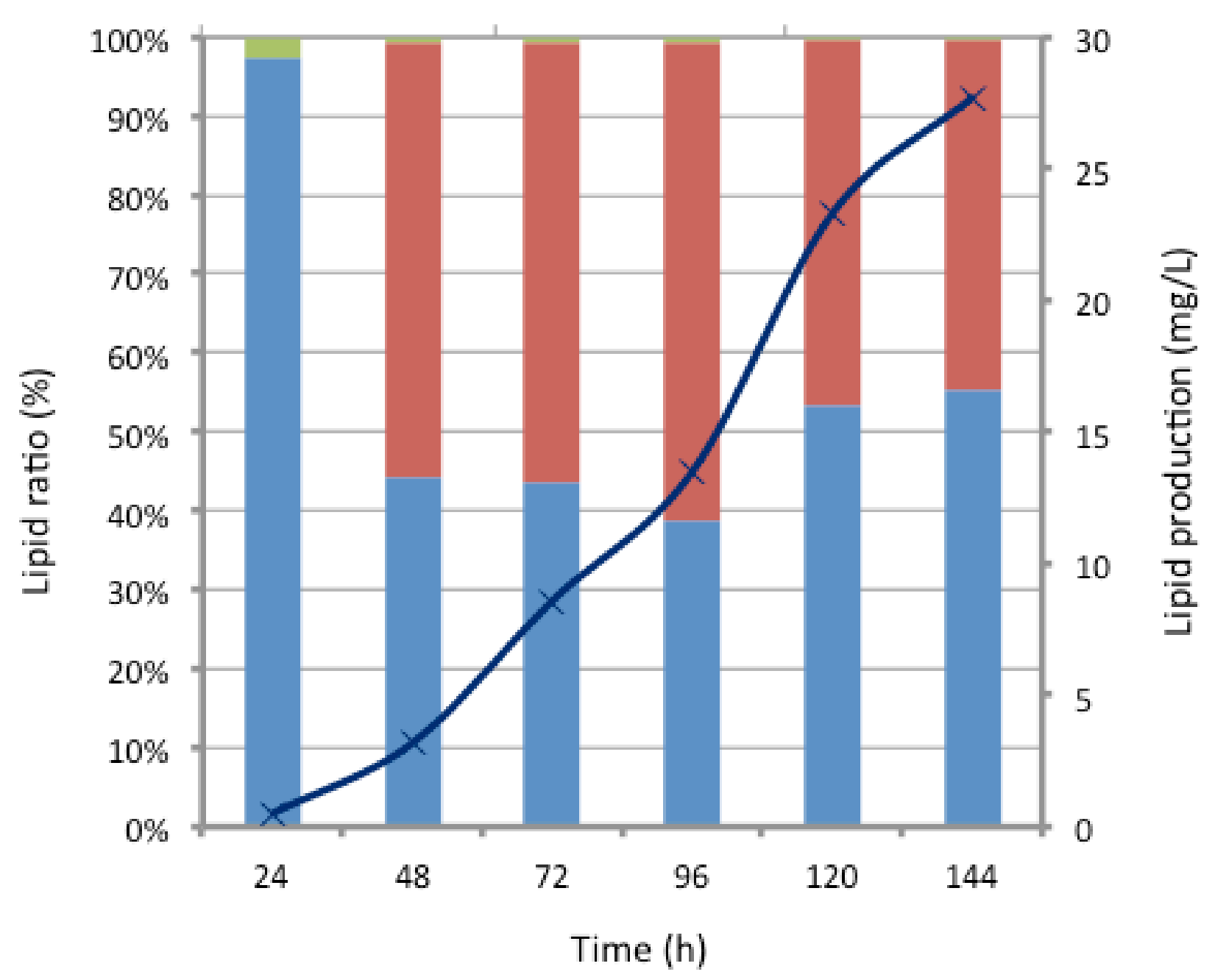

Fig. 3 
Table 1 Conversion rates from carbon sources into total lipids.

\begin{tabular}{lccccc}
\hline Carbon source & $\begin{array}{c}\text { Consumption of } \\
\text { VFA }(\mathrm{mM})\end{array}$ & $\begin{array}{c}\text { Total carbon } \\
(\mathrm{mg} / \mathrm{L})\end{array}$ & $\begin{array}{c}\text { Total lipid } \\
(\mathrm{mg} / \mathrm{L})^{\mathrm{a}}\end{array}$ & $\begin{array}{c}\text { Total lipid } \\
(\mathrm{mg} / \mathrm{g}-\mathrm{DCW})^{\mathrm{a}}\end{array}$ & $\begin{array}{c}\text { Carbon yield of } \\
\text { total lipids }(\%)\end{array}$ \\
\hline Acetic acid & 15.43 & 926.57 & 4.3 & 21.3 & 0.46 \\
Propionic acid & 5.06 & 374.84 & 2.4 & 11.2 & 0.65 \\
Butyric acid & 3.28 & 289.00 & 3.9 & 8.7 & 1.35 \\
Valeric acid & 9.81 & 1001.89 & 2.0 & 3.4 & 0.20 \\
Glycerol & 6.68 & 615.18 & 3.8 & 14.7 & 0.61 \\
\hline
\end{tabular}

a: Total lipid was extracted by hexane. 
Table 2 Accumulation of lipids by Nitratireductor sp. OM-1 grown under the nitrogen- depletion conditions .

\begin{tabular}{ccccccc}
\hline $\begin{array}{c}\text { Concentration of } \\
\mathrm{NH}_{4} \mathrm{Cl}\end{array}$ & $\begin{array}{c}\text { Total lipid/DCW } \\
(\mathrm{mg} / \mathrm{g})^{\mathrm{a}}\end{array}$ & $\begin{array}{c}\text { Total FA/DCW } \\
(\mathrm{mg} / \mathrm{g})^{\mathrm{a}}\end{array}$ & $\begin{array}{c}\text { Pentadecenoic acid } \\
(\mathrm{mg} / \mathrm{g})^{\mathrm{b}}\end{array}$ & $\begin{array}{c}\text { Palmitoleic acid } \\
(\mathrm{mg} / \mathrm{g})^{\mathrm{b}}\end{array}$ & $\begin{array}{c}\text { Stearic acid } \\
(\mathrm{mg} / \mathrm{g})^{\mathrm{b}}\end{array}$ & $\begin{array}{c}\text { Linoleic acid } \\
(\mathrm{mg} / \mathrm{g})^{\mathrm{b}}\end{array}$ \\
\hline $20 \mathrm{mM}$ & 6.36 & 2.92 & 0.73 & 0.52 & 0.07 & 0.90 \\
$10 \mathrm{mM}$ & 7.75 & 4.88 & 1.11 & 1.14 & 1.11 & 1.61 \\
$1 \mathrm{mM}$ & 246.79 & 10.83 & 2.04 & 2.44 & 0.24 & 3.77 \\
$0.1 \mathrm{mM}$ & 209.96 & 23.4 & 6.34 & 6.13 & 0.20 & 5.07 \\
$0 \mathrm{mM}$ & 705.79 & 26.73 & 5.36 & 7.28 & 0.48 & 9.10 \\
\hline
\end{tabular}

a; Total lipid was extracted by chloroform : methanol (1:1).

b; Total FA was extracted by $10 \% \mathrm{HCl}$-methanol and methyl esterified. 